\title{
Experiencia de reconversión de un servicio clínico anestésico-quirúrgico en unidad de pacientes críticos COVID-19 en un hospital universitario
}

\section{Experience of transforming a clinical surgical-anesthetic unit into a COVID-19 critical patient one in a teaching hospital}

María Mercedes Aguirre MD. ${ }^{1, *}$, Felipe Maldonado MD. ${ }^{1}$, Paula Fuenzalida MD. ${ }^{1}$, Roberto González MD. ${ }^{1}$, Nicolás Moreno², Ignacio Lalanne2, Patricia Bisbal EU ${ }^{3}$, Marcela Cifuentes MD. ${ }^{4}$, Francisco Gonzáles MD. ${ }^{5}$, Darwin Phillips ${ }^{6}$, Marioli Lazo7 , Jaime Escobar MD. ${ }^{8}$, Nivia Estuardo MD. ${ }^{9}$

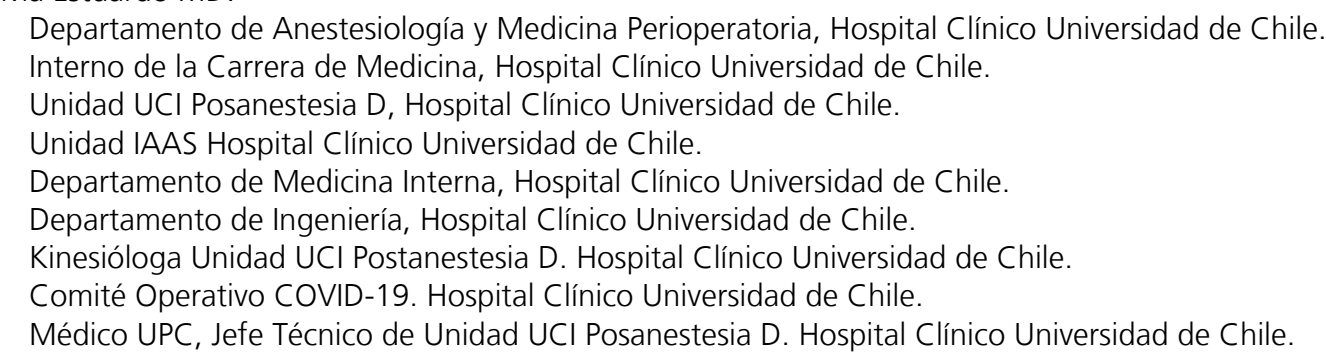

No hay financiamiento para este artículo.

Los autores declaran ausencia de conflictos de interés.

Fecha de recepción: 01 de junio de 2021 / Fecha de aceptación: 04 de junio de 2021

\begin{abstract}
Introduction: The experience of restructuring a clinical surgical-anesthetic unit into a critical patient unit in charge of surgicalanesthetic personnel is presented during the period from May to July 2020 in the context of a SARS-CoV-2 pandemic. Objectives: Describe the unit's restructuring process, considering technical aspects, changes in staff functions, clinical outcomes of the patients, quality indicators obtained and the psychological impact on the healthcare team. Matherial and Methods: The strategies implemented by the responsible experts were described (ie: engineering). Clinical data were obtained from an institutional database and electronical medical records. The management of human resources was described using administrative records of the services of anesthesiology, OR and critical patient unit. The psychological impact on the unit staff was evaluated by applying the Maslach questionnaire. The quality of the clinical management of the unit was obtained from the compilation of standardized quality indicators for the critical patient units of the institution. Results: 25 patients were admitted in the unit. The mean age was $62 \pm 12$ years. About the complications, 52\% had pulmonary embolism, 36\% had acute kidney injury, and 1 patient died. The prevalence of Burnout Syndrome was $73.6 \%$. The occurrence of adverse events was minimal. Discussion: The transformation of an anesthetic-surgical unit into a COVID critical patient one, demands a complex net of coordinated strategies to allow facing the attention demand with positive clinical results, at the expense of the health care team mental health.
\end{abstract}

Key words: Conversion of critical beds, COVID-19 pandemia, burnout in health care team, critical patient units converted, anesthesiology unit converted, COVID critical care unit.

mmaguirre@uchile.cl

*ORCID: https://orcid.org/0000-0001-6703-9493 


\section{RESUMEN}

Introducción: Se presenta la experiencia de reconversión de una unidad de cuidados posanestésicos a una unidad de cuidados intensivos a cargo de personal anestésico-quirúrgico entre mayo y julio de 2020, en contexto de pandemia por SARS-CoV-2. Objetivos: Describir el proceso de reconversión considerando aspectos técnicos, pertinentes al recurso humano, resultados clínicos, indicadores de calidad e impacto psicológico en el equipo de salud. Materiales y Métodos: Se describen las estrategias implementadas por los expertos responsables. Se obtienen datos clínicos desde base de datos institucional y ficha clínica electrónica. Se describe la gestión del recurso humano utilizando registros administrativos de los servicios involucrados. El impacto psicológico en el personal fue evaluado aplicando el cuestionario de Maslach. La calidad de la gestión clínica se obtiene a partir de indicadores de calidad estandarizados para las unidades de pacientes críticos de nuestro establecimiento. Resultados: Se atendieron 25 pacientes en la unidad. La edad promedio fue $62 \pm 12$ años. El 52\% presentó tromboembolismo pulmonar, 36\% injuria renal aguda como complicación. Un paciente falleció. La prevalencia de síndrome de Burnout fue de 73,6\%. La ocurrencia de eventos adversos fue baja. Conclusiones: La reconversión de una unidad anestésico-quirúrgica a una unidad crítica COVID-19, demanda un complejo entramado de estrategias coordinadas que permiten responder a la demanda de atención con resultados clínicos positivos, a expensas del costo de la salud mental del equipo de salud involucrado.

Palabras clave: Reconversión camas críticas, pandemia COVID-19, burnout en equipos de salud, unidades de pacientes críticos reconvertidas, unidades de anestesiología reconvertidas, unidades críticas COVID-19.

\section{Introducción}

E Hospital Clínico de la Universidad de Chile (HCUCH), es un hospital universitario de carácter estatal, cuya visión y misión apuntan "...a la formación de profesionales de la salud de excelencia, la investigación y la mejor práctica clínica, que otorgue a la comunidad soluciones integrales de salud"[1]. A finales de 2019 se descubrió nueva cepa del virus coronavirus, el SARS-CoV-2, el cual desató una pandemia que persiste hasta el día de hoy, produciendo la enfermedad por coronavirus (coronavirus desease - COVID-19). Dentro de sus manifestaciones destacan la insuficiencia respiratoria aguda y una respuesta inflamatoria sistémica grave, entre otras, lo cual generó un importante aumento en la demanda de camas críticas en el país. En este contexto, el HCUCH cumpliendo con su compromiso social, desarrolló un plan estratégico, contemplando la reconversión de la unidad de posanestesia $D(P A D)$ en una unidad de cuidados intensivos $(\mathrm{UCI})$, destinando el uso de recursos físicos y humanos, implicando el cese de sus tareas quirúrgicas habituales según el mandato de la autoridad sanitaria expuesto en el Decreto oficial el 25 de marzo de 2020[2].

El objetivo de este artículo es describir este proceso de reconversión considerando los aspectos técnicos, los resultados clínicos de los pacientes atendidos, los indicadores de calidad obtenidos en la unidad y el impacto psicológico en el equipo de salud participante.

\section{Desarrollo}

A la fecha, se ha reportado que $15 \%$ de quienes desarrollan la infección por SARS-CoV-2 requiere hospitalización y en $6 \%$ a $12 \%$ hospitalización en una unidad de paciente crítico (UPC)[3],[4],[5]. El HCUCH es un establecimiento tipo uno de alta complejidad con 504 camas totales, 22 camas de cuidados intensivos (UCI) y 59 camas de tratamiento intermedio (UTI).

Durante el período de la pandemia 2020 por COVID-19, en respuesta a la demanda asistencial, fueron reconvertidas camas y servicios, aumentando la oferta de camas de UCI y UTI a 59 cada una. Esto significó un complejo trabajo de planificación dado las características microbiológicas del SARS-CoV-2 y su comportamiento epidemiológico. A continuación, se describe el proceso de reconversión de una unidad de cuidados posanestésicos a UCI-COVID-19 (PAD-UCI), la cual abrió el día 14 de mayo de 2020 y cerró el 26 de julio del mismo año.

\section{Infraestructura}

El HCUCH cuenta con tres áreas de pabellones quirúrgicos distribuidos en tres sectores diferentes del hospital, cada uno con su unidad de recuperación posanestésica correspondiente. La unidad de recuperación PAD es la de mayor capacidad, y de funcionamiento continuo ya que en dicho sector se desarrolla la actividad quirúrgica de urgencia. Se definió la utilización de este espacio para albergar pacientes críticos COVID-19 por las siguientes razones:

1. Ser un espacio cerrado favoreciendo las condiciones de seguridad necesarias para evitar contagios.

2. Contar con instalaciones adecuadas para el funcionamiento de equipos requeridos para la atención de estos pacientes, esto es, red eléctrica, de gases clínicos y de aspiración apropiadas.

3. Contar con espacios físicos de trabajo y descanso seguros; estar próximo a los pabellones quirúrgicos en que se resolverían patologías quirúrgicas urgentes en pacientes COVID-19 y donde se realizarían las intubaciones orotraqueales (IOT) de estos mismos pacientes, facilitando la ayuda en caso necesario frente a complicaciones del procedimiento, ya que los médicos a cargo de la unidad serían anestesiólogos.

Las observaciones técnicas consideradas para habilitar este espacio contiguo al quirófano COVID-19 se detallan a continuación.

Un aspecto relevante para considerar fue la vía de contagio del SARS-CoV-2, para lo que en base a la evidencia científica publicada hasta ese entonces y las recomendaciones de expertos internacionales quienes, en julio de 2020, advierten sobre la transmisión del virus a través del aire en forma de aerosoles[6], que posteriormente es avalada por organizaciones tales como 
la OMS, NHS, CDC y MINSAL[3],[7],[8],[9],[10],[11]. Lo anterior, fundamentado en que las personas emiten gotas y aerosoles al toser, estornudar, hablar, así como al ser sometidos a procedimientos generadores de aerosoles (PGA) (IOT, aspiración de secreciones traqueales, entre otros)[12],[13],[14]. Así, se asumió que al interior de los pabellones quirúrgicos destinados a la atención de pacientes COVID-19 y en la unidad de PAD$\mathrm{UCl}$, existiría una elevada producción de aerosoles, exigiendo su control por medio de medidas de ventilación descritas más adelante.

Respecto de las gotitas y aerosoles, estas son partículas diferenciadas por su tamaño, por la distancia que pueden alcanzar respecto del emisor y por el tiempo en que se mantienen en suspensión[15]. Las partículas de tamaño mayor a 5 micras (gotitas, grandes) al caer tienden a describir trayectorias balísticas (parabólicas, determinadas por la aceleración de gravedad), mientras que las partículas menores de 5 micras (aerosoles, pequeñas) se mantienen más tiempo suspendidas en el aire, dado su reducido diámetro y masa (Ley de Stokes)[16],[17]. Así, las gotitas caen dentro de un radio aproximado de 1,5 metros, mientras que los aerosoles se mantienen en suspensión y se desplazan prácticamente junto con el aire que las rodea. Adicionalmente, por evaporación, las partículas grandes se pueden transformar en pequeñas, incorporándose desde el nivel del suelo como aerosol aerotransportado. De allí que para reducir el riesgo de contagio toma relevancia que el aire contaminado aerosolizado deba ser extraído de la forma más directa y unidireccional posible, con dilución máxima y reemplazado por aire exterior limpio.

\section{Flujos de aire limpio exterior y extracción de aire contaminado}

En los pabellones quirúrgicos, las normas de ventilación identifican al medio externo como la fuente de contaminación, siendo el aire filtrado y renovado previo a su ingreso al quirófano, reduciendo los contaminantes por medio de barreras físicas y de dilución. Asimismo, para que la contaminación en los pasillos adyacentes al quirófano no ingrese a éstos, el quirófano debe contar con presión positiva (PP).

En pacientes hospitalizados, con agentes con vías de transmisión por gotas y aerosoles, la fuente principal de contaminación deja de ser el exterior de la unidad del paciente (UP) y el paciente pasa a ser la fuente contaminante; debiendo así la UP contar con presión negativa (PN), de modo que el aire contaminado sea direccionado al exterior de la UP, evitando el flujo hacia unidades adyacentes (Figura 1).

Puesto que la PP en la ventilación de los pabellones quirúrgicos es estructural y no modificable en el corto plazo, se adoptaron las siguientes medidas para minimización de riesgos en el uso de pabellones quirúrgicos con pacientes con COVID-19:

1. Mantención del PP en cada quirófano, para maximizar la dilución del aerosol emitido por los pacientes.

2. Agrupar los pabellones asignados a pacientes con COVID-19 y segmentar sus pasillos receptores de la PP que originan flujo de aire contaminado (pero diluido) a través de sus puertas.

3. Instalar un sistema de extracción de aire con flujos de 6.000 $\mathrm{m}^{3} / \mathrm{h}$ desde los pasillos hacia el exterior, así generar la mínima ruta interior del aire contaminado y una ruta unidireccional.

Paralelamente, la PAD-UCl en que los pacientes permanecían hospitalizados, es un recinto que cuenta con inyección y extracción de aire (sin filtro absoluto).

Con el objeto de evitar riesgos de reflujo de aire contaminado hacia PAD-UCI se establecieron medidas destinadas a evitar las distintas categorías de riesgo:

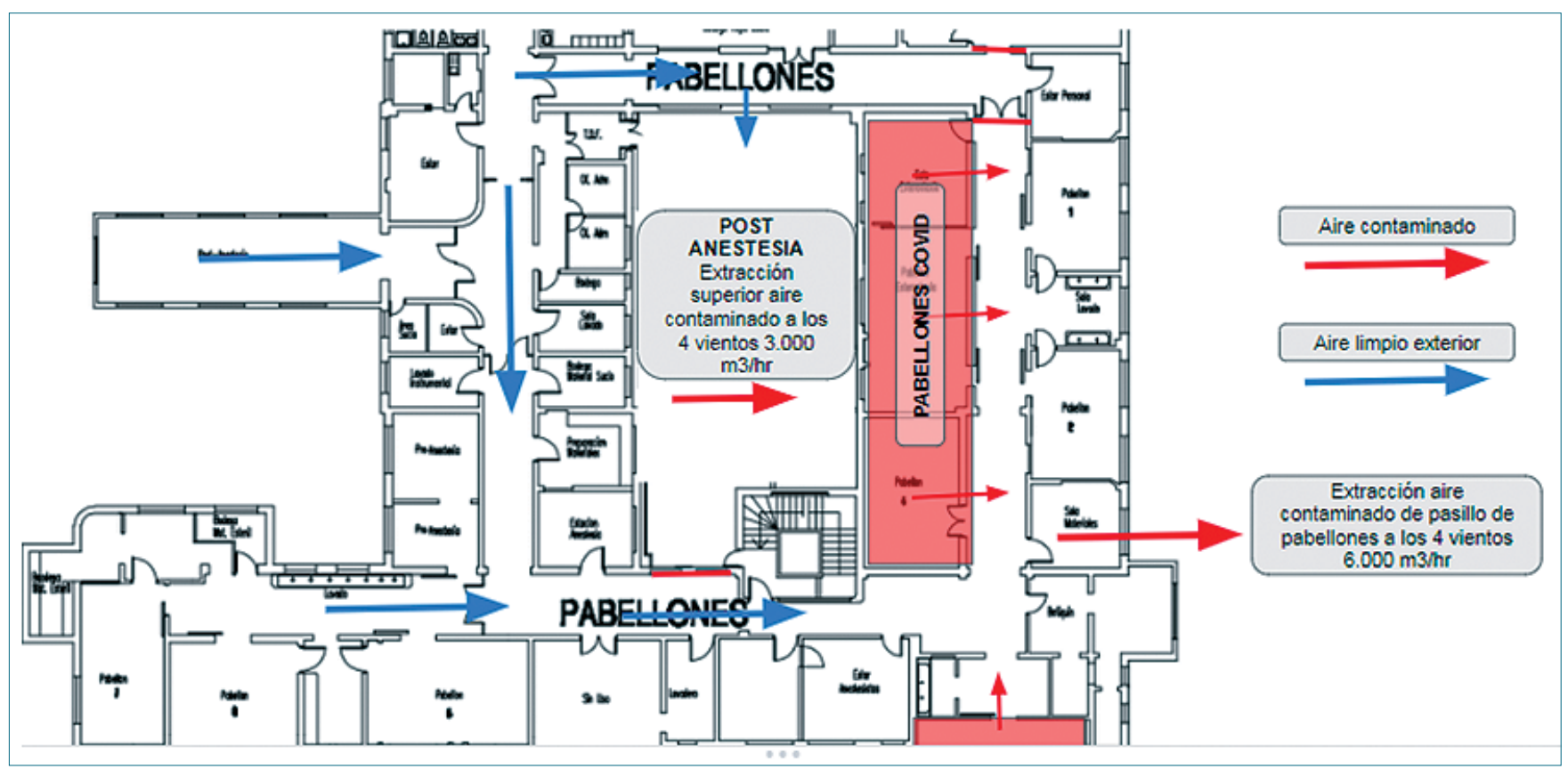

Figura 1. 
- Riesgo de reflujo por balance de las presiones negativas de extracción entre el recinto de posanestesia y el pasillo de pabellones COVID-19: Se apagó la inyección de aire exterior por cielo hacia PAD-UCI permitiendo el ingreso de aire exterior a nivel de puertas, con barrido piso-cielo; y se mantuvo la extracción del aire contaminado de ese recinto con caudal de $3.000 \mathrm{~m}^{3} / \mathrm{h}$, maximizando la dilución.

- Riesgo de reflujo desde el pasillo de pabellones COVID-19 a puerta norte de posanestesia: Se bloqueó el pasillo de pabellones COVID-19, de modo que la presión negativa no compitiera con el acceso de aire exterior limpio al recinto de PAD-UCI por la puerta norte, asegurando la máxima dilución y efectividad de la extracción propia de ese recinto. A su vez, el recinto de posanestesia cuenta con una puerta automática normalmente cerrada, que se abre por sensor de necesidad de paso.

- Riesgo de reflujo desde el pasillo de pabellones COVID-19 a puerta sur de posanestesia: El pasillo sur hacia los pabellones COVID-19 se mantuvo abierto al paso, por lo que se bloqueó el acceso sur hacia PAD-UCl, con el fin de no competir con el ingreso de aire limpio exterior a nivel de puerta y asegurar la máxima eficacia extractiva del aire contaminado del recinto de PAD-UCI.

\section{Medidas de prevención y control de infecciones asociadas a la atención en salud (IAAS) destinadas a prevenir el contagio del personal}

Se realizó un protocolo de seguridad microbiológico orientado a las exposiciones presentes en PAD-UCI, elaborado por un equipo multidisciplinario conformado por la jefatura de unidad, equipo de enfermería, miembros de UPC, Dirección del establecimiento, equipo de Ingeniería y mantención, y el Comité de IAAS del establecimiento.

\section{Organización planta física}

El grupo de trabajo aprobó las medidas establecidas para la planta física por el equipo de ingeniería, recientemente detalladas.

Se definió mantener acceso restringido al sector donde se encuentra la PAD-UCI y los pabellones quirúrgicos COVID-19, incluyendo la zona de casilleros, entrega de vestuario y baños, para personal reconvertido destinado a UPC-COVID-19. Se estableció no usar el resto de los quirófanos para cirugías de pacientes no COVID-19.

La planta física de la unidad consiste en un gran espacio aislado de los pasillos, sin separación mecánica entre las unidades de pacientes, ni entre éstas y las áreas de estación de enfermería y administrativas. Por esta razón se dispuso que el personal debía permanecer en la unidad usando permanentemente los elementos de protección personal (EPP), manteniendo la mínima circulación hacia fuera de ésta. La capacidad habitual de esta recuperación es de 12 pacientes. Para resguardar una distancia mínima de 1,5 metros entre pacientes, se establecieron 6 cupos operativos.

Se establecieron las siguientes áreas de trabajo dentro de la unidad:

- Estación de enfermería con sus áreas limpia, sucia y administrativa.
- Área de trabajo sucio (lavamanos, lavadero, bota aguas, basura, ropa sucia) habilitado en un sector fuera de la unidad.

- Los residuos del establecimiento de atención en salud (REAS) se mantuvieron donde funcionaba en el pabellón normalmente.

- Área administrativa para trabajo de médicos.

- Se mantuvo una sola puerta de acceso de apertura automática.

- Se definieron las áreas para descanso, alimentación y baños, fuera de la unidad, pero dentro del mismo sector de pabellones del HCUCH.

\section{Elementos de protección personal (EPP)}

Dado el espacio común abierto compartido entre pacientes, estación de enfermería y áreas administrativas, se estimó que el riesgo de permanencia de gotitas y aerosoles en suspensión podría ser mayor. Por ello se definió que todo el personal debía acceder al mayor nivel de EPP disponible en el momento, según las recomendaciones internacionales y el protocolo interno[18].

Se estableció el uso de EPP en 3 capas para la protección de tronco y extremidades, y mascarilla de tipo N95 (NIOSH) de uso extendido. Primera y segunda capa de uso obligatorio dentro de PAD-UCl, tercera capa para uso exclusivo individual con pacientes COVID-19.

- Vestuario:

- "Primera capa": traje clínico institucional.

- "Segunda capa": delantal quirúrgico de aislamiento (FDA clase II) de manga larga de único uso sobre la primera capa y guantes estériles de uso permanente.

- "Tercera capa": Delantal plástico de manga larga y guantes de procedimientos desechables sobre el delantal quirúrgico para reducir la contaminación por gotitas o contacto al tener contacto con el paciente y su unidad. Esta capa era retirada inmediatamente posterior a salir de la unidad del paciente.

- Para la protección de la cabeza y cuello se utilizó una capucha de confección local sin cobertura de cara.

- Para la protección de la mucosa ocular, nasal y orofaríngea, se otorgó el uso extendido de mascarilla N95 con hasta 24 h de duración. Esta podía dejarse en un contenedor exclusivo de uso individual mientras no fuera utilizada. En caso de uso de mascarilla N95 durante PGA se indicaba su desecho inmediato y reemplazo. Además, sobre las máscaras se utilizó escudo facial de plástico trasparente.

- Para la realización de PGA se asignó a la unidad el uso de máscaras elastoméricas de rostro completo $3 \mathrm{M}^{\circledR}$ con filtro P100 incorporado. Los PGA, fueron protocolizados por el comité de IAAS de la institución de acuerdo con la evidencia vigente[18],[19], y debían realizarse en un quirófano de uso exclusivo.

Para todos los funcionarios, previo al egreso de la PAD-UCl, se estableció el retiro de la segunda capa y guantes en zona contigua a puerta de salida, y posterior higienización de manos. Fuera de la unidad, se realizaba el retiro de mascarilla, escudos faciales y capuchas, que eran eliminadas en contenedores de residuos biológicos. Finalmente se repetía la higienización de manos.

El personal de la unidad estuvo bajo vigilancia epidemioló- 
gica frente a la ocurrencia de casos de COVID-19 intrahospitalarios.

\section{Flujo de insumos y otros}

Se restringió el flujo de insumos y elementos (teléfonos móviles $u$ otros objetos de uso personal) desde y hacia la unidad. Toda solicitud de algún elemento hacia fuera de la unidad se canalizó a través de un auxiliar de servicio, quien gestionaba y dejaba el elemento en la puerta. Los documentos físicos (indicaciones y recetas) eran de uso exclusivo de la unidad. Dentro de la unidad se contó con computadores suficientes para las tareas administrativas del personal, teléfono fijo habilitado para uso interno y particular y un ecógrafo exclusivo de la unidad.

Los pacientes sólo salieron de la unidad en caso de estricta necesidad para realizar exámenes radiológicos, intervenciones o ser extubados en pabellones quirúrgicos.

\section{Recurso humano}

El recurso humano necesario para implementar la PAD-UCI fue gestionado convirtiendo funciones de personal a partir de tres servicios: UPC, pabellones quirúrgicos y anestesiología. El servicio de pabellones quirúrgicos proveyó de enfermeros/as (EU), auxiliares de enfermería (AE) y auxiliares de servicio (AS).

La fracción del personal de pabellones quirúrgicos que reconvirtió sus funciones para trabajar en PAD-UCI fue: 15\% de EU (27), 6\% de AE (106) y $15 \%$ de AS (40).

Durante el funcionamiento de la unidad se presentaron 6 licencias médicas de funcionarios: 1 AS 2 AE y 4 EU. Sólo una correspondió a contagio por SARS-CoV-2, atribuido a contacto externo.

Del servicio de anestesiología, 14/35 especialistas reconvirtieron sus funciones voluntariamente para ejercer en esta unidad. La cobertura fue a jornada completa y se distribuyeron 3 pacientes por médico. Hubo participación ocasional de residentes de anestesiología.

El número de licencias por COVID-19 entre el personal médico-anestesiólogo fueron 3, dos por ser contactos estrechos intradomiciliarios y una por contagio directo. No hubo licencias por otras causas.

Un EU del servicio de anestesiología participó inicialmente en la unidad, pero posteriormente fue reasignado a otra. $14 \mathrm{AE}$ reconvirtieron sus labores a la unidad, 3 de ellos presentaron licencia por contagio COVID-19; dos por contacto estrecho y uno por contagio, sin determinar origen.

Dos veces al día, un médico intensivista visitó la unidad, llevando la continuidad del manejo de los casos y discutiendo las decisiones clínicas junto a los anestesiólogos. Todo paciente recibió atención kinésica respiratoria y motora dos veces al día. La necesidad de atención por otro profesional fue gestionada en forma telefónica.

\section{Resultados clínicos de pacientes de la unidad}

Un total de 25 pacientes fueron atendidos en la unidad PAD-UCI durante 73 días. La edad promedio fue de $62 \pm 12$ años (DS). Las comorbilidades, complicaciones, y días en apoyo ventilatorio de los pacientes se detalla en la Tabla 1.

El $52 \%$ de los pacientes evolucionó con tromboembolismo

\begin{tabular}{|c|c|}
\hline \multicolumn{2}{|c|}{ Tabla 1} \\
\hline Número de pacientes & 25 \\
\hline Edad $(n \pm s d)$ & $62 \pm 12$ \\
\hline Comorbilidades & $\mathrm{n}(\%)$ \\
\hline HTA & $14(56)$ \\
\hline Obesidad & $10(40)$ \\
\hline Diabetes mellitus & $9(36)$ \\
\hline Tabaquismo & $4(16)$ \\
\hline C Coronaria & $2(8)$ \\
\hline EPOC & $2(8)$ \\
\hline Asma & $1(4)$ \\
\hline Complicaciones & $\mathrm{n}(\%)$ \\
\hline Tiempo desde diagnóstico (PCR +) & $11 \pm 10$ días \\
\hline TEP & $13(52)$ \\
\hline AKI & $9(36)$ \\
\hline \multicolumn{2}{|l|}{ Injuria miocárdica } \\
\hline \multicolumn{2}{|l|}{ Infarto } \\
\hline Muerte & $1(4)$ \\
\hline Ventilación & Días \\
\hline Promedio días pre VM & $5 \pm 5$ días \\
\hline Promedio días VMI & $30 \pm 24$ días \\
\hline \multirow[t]{2}{*}{ Promedio días VMI + prono } & $7 \pm 4$ días \\
\hline & n (\%) \\
\hline Número pacientes en CNAF & $18(75)$ \\
\hline $\begin{array}{l}\text { Número pacientes en CNAF-prono } \\
\text { vigil }\end{array}$ & $7(28)$ \\
\hline Número de pacientes en VMI & $25(100)$ \\
\hline \multicolumn{2}{|l|}{$\begin{array}{l}\text { Número de pacientes en VMI- } \\
\text { prono* }\end{array}$} \\
\hline Al menos un ciclo de prono & $17(68 \%)$ \\
\hline
\end{tabular}

pulmonar y $36 \%$ (9 pacientes) con injuria renal aguda. De ellos, uno requirió terapia de reemplazo renal. El 75\% de los pacientes ingresaron en ventilación mecánica luego de 5 días en promedio de uso de cánula nasal de alto flujo. Sólo un paciente ingresó usando CNAF luego de haber completado un período de VMI para ser trasladado a otra unidad. Los pacientes permanecieron en promedio $30 \pm 24$ días en ventilación mecánica, llegando a un máximo de 70 días de VMI en la unidad. Un paciente falleció luego de 41 días de ventilación mecánica invasiva.

\section{Resultados de calidad y seguridad de la unidad}

No se establecieron instrumentos destinados a medición de satisfacción usuaria, sin embargo, a la fecha no hay registro de reclamos hacia la institución con relación a atención de pacientes en la unidad. Respecto a los eventos registrados la información es la siguiente:

- Eventos adversos: 1, lesión por presión que fue catalogada al ingreso a la unidad tipo 2 y evolucionó durante su estadía a tipo 4. 
- Casi evento: 3, un error de medicación, uno relacionado con planta física y uno con equipamiento.

- Incidentes: 2, desplazamientos accidentales de tubo-orotraqueal que requirieron reintubación.

- Eventos centinelas: no hubo.

Tras el cierre de unidad PAD-UCI, se realizó un análisis retrospectivo en formato "Debriefing" entre los profesionales que trabajaron en la unidad, respecto de los niveles de seguridad y calidad de funcionamiento del servicio. Tras esto destaca una apreciación general de éxito dado la complejidad de los pacientes atendidos, la carga asistencial por persona, personal inexperto, equipos de trabajo nuevos, condiciones de trabajo (uso de EPP por períodos extendidos).

El trabajar en una unidad de alta complejidad y en ambiente adverso, pudo haber redoblado los esfuerzos en términos de prevención y cuidados, lo cual se reflejó en los resultados por un lado y en el cansancio de personal paramédico y enfermeros/as.

\section{Consecuencias en salud emocional en el personal participante}

Dado el contexto de reconversión de unidades y el aumento de la complejidad de atención que debió prestar el personal de PAD-UCl, se estableció la premisa de que el cambio en funciones habituales debió tener impacto psicológico en quienes participaron. Por ello, se aplicó una encuesta validada para la evaluación de la presencia del síndrome de Burnout en el personal del HCUCH pertenecientes a unidades anestésico-quirúrgicas que participaron de las distintas unidades de reconversión del HCUCH durante los meses de marzo-agosto de 2020. Se recibieron 47 encuestas válidas entre el 10 de noviembre del año 2020 y el 26 de enero del año 2021, de las cuales 19 corresponden a personas que participaron del funcionamiento de la PAD-UCI.

Las Tablas 3 y 4 resumen las características sociodemográficas y profesionales de los participantes.

Durante los meses de marzo a agosto, 31,6\% de los participantes estuvo en cuarentena al menos una vez. El $47 \%$ tuvo que hacer cambios en su núcleo familiar por el riesgo de contagio. El 100\% cambió el rol que tenía en su trabajo previo a la pandemia, ninguno se desempeñaba en UCl. Todos modificaron su horario habitual de trabajo, la mayoría tenía exclusivamente horario diurno. El 78,9\% de los participantes refieren un aumento de la carga laboral durante este período.

Respecto a la calidad de vida, el $68 \%$ refiere que disminuyó su tiempo de ocio; el $84,2 \%$ disminuyó sus horas de sueño promedio; en cuanto a alimentación, el 78,9\% refiere que su alimentación se tornó menos saludable; el 63,1\% incrementó su consumo de alcohol.

Al indagar respecto a las necesidades actuales, primaron las respuestas de disminución de la carga laboral y "otra", destacando en la descripción libre la necesidad de vacaciones y tiempo libre. En menor preponderancia se seleccionó el apoyo psicológico y la opción "no creo necesitar nada adicional". El $31,6 \%$ sintió deseos de renunciar a su trabajo. De las preocupaciones, primó el miedo a contagiar a algún miembro de la familia.

En cuanto a los elementos percibidos como positivos du-

\begin{tabular}{lcc}
\multicolumn{3}{l}{ Tabla 2. Características sociodemográficas de los participantes } \\
\hline Características sociodemográficas & $\%$ & $\mathbf{n}$ \\
Profesión & & \\
Enfermera(o) & 5,3 & 1 \\
Técnica(o) en enfermería nivel superior & 36,8 & 7 \\
Médica(o) Staff HCUCH & 47,4 & 9 \\
Médica(o) residente HCUCH & 10,5 & 2 \\
Rango de edad & & \\
21 a 29 años & 15,8 & 3 \\
30 a 39 años & 31,6 & 6 \\
40 a 49 años & 42,1 & 8 \\
50 a 59 años & 10,5 & 2 \\
Más de 60 años & 0 & 0 \\
Género & & \\
Femenino & 63,2 & 12 \\
Masculino & 36,8 & 7 \\
Hijos & & 7 \\
Sí & 63,2 & 12 \\
No & 36,8 & 7 \\
\hline
\end{tabular}

\begin{tabular}{lcc}
\multicolumn{2}{c}{ Tabla 3. Características profesionales de los participantes } \\
\hline Características profesionales & $\%$ & $\mathbf{n}$ \\
Experiencia en UCI & 21,1 & 4 \\
1 a 5 años & 10,5 & 2 \\
6 a 10 años & 10,5 & 2 \\
Menos de 1 año & 57,9 & 11 \\
Sin experiencia previa & & \\
Experiencia profesional & 15,8 & 3 \\
1 a 5 años & 21,1 & 4 \\
6 a 10 años & 10,5 & 2 \\
11 a 15 años & 26,3 & 5 \\
16 a 20 años & 26,3 & 5 \\
Más de 20 años & & \\
Preparación previa & 78,9 & 15 \\
Sí & 21,1 & 4 \\
No & & 6 \\
Suficiente & 47,4 & 9 \\
Sí & 31,6 & \\
No & & \\
\hline
\end{tabular}

rante el trabajo en la PAD-UCI destacan, en orden decreciente, la relación de cooperación construida entre los distintos estamentos, además de sentir que se estaba contribuyendo a la posibilidad de mejoría de pacientes graves y la percepción que se estaban haciendo las cosas bien en la unidad. Por su parte, 
los elementos más difíciles de sobrellevar en la unidad fueron: la utilización de EPP por períodos prolongados, trabajar en un lugar aislado en dependencias diferentes y el número de pacientes en decúbito prono que requerían atención clínica compleja.

En cuanto a la disposición de participar en una unidad similar, 16\% lo haría sin mayores cuestionamientos, $21 \%$ no estaría dispuesto a hacerlo en ninguna circunstancia. El resto (63\%) lo haría sólo de manera condicional.

Para la evaluación de presencia de Burnout, se utilizó la escala de Maslach validada en idioma español, en formato digital. Se determinó como criterio diagnóstico de síndrome de Burnout tener 2 de 3 dimensiones afectadas de la escala de Maslach. Se pesquisó presencia de síndrome de Burnout en el $73,6 \%$ de los participantes. El desglose de los resultados por dimensión afectada se encuentra en la Tabla 3.

En cuanto a la valoración estadística, al evaluar factores de riesgo conocidos para Burnout, como la carga laboral excesiva, el número de hijos, los cambios en la actividad cotidiana o, inclusive, al analizar las características sociodemográficas del grupo participante, no se encontró una asociación estadísticamente significativa entre estos elementos y los resultados obtenidos de la escala de Maslach.

\section{Discusión}

La experiencia de reconversión de una unidad implica un enorme desafío institucional para el equipo de salud e individual, más aún cuando esta es inesperada y requiere de una rápida adecuación a las características de un agente no del todo comprendido en dicha fecha.

Dentro de esta estrategia de reconversión se abordaron en formar multidisciplinaria y colaborativa diferentes aspectos intentando resguardar tanto la seguridad de los pacientes como del personal de salud, en un momento en que la información respecto a la propagación del virus estaba en desarrollo.

Respecto al resultado clínico de los pacientes atendidos en esta unidad y considerando la atención brindada por personal no experto en el área crítica, destaca una baja tasa de complicaciones médicas asociadas a la atención y baja tasa de mortalidad (4\%) con respecto a las series internacionales, que reportan valores entre $25,3 \%$ y $85 \%[20],[21],[22],[23]$. El porcentaje de mortalidad en el total de pacientes ventilados en todas las UPC instaladas en el HCUCH durante el año 2020 fue de $25 \%$. Destaca también la ocurrencia de escasos eventos negativos asociados a la calidad y seguridad en la unidad.

Es destacable la efectividad de las medidas de protección personal que se adoptaron y su correcta implementación, ya que el porcentaje de contagios del personal de la unidad PAD$\mathrm{UCI}(7 \%)$ se encontró dentro del rango en relación al reportado en series internacionales (3,4\% a 19\%)[24],[25],[26],[27],[28],[ 29],[30].

Sin embargo, es importante considerar el impacto psicológico negativo en el personal de la unidad, correlacionado con el aumento de carga laboral, la modificación del tipo de actividades laborales habituales y la mayor exposición a estresores (trabajo con alto riesgo de contagio microbiológico, jornada laboral extensa, adecuaciones actividades extralaborales, conformación de nuevos grupos de trabajo). Lo anterior evidenciado
Tabla 4. Indicio de síndrome de Burnout en subescalas de Maslach

\begin{tabular}{lc}
\hline Dimensión & Indicios de síndrome de Burnout n (\%) \\
Cansancio emocional & $10(52,6)$ \\
Despersonalización & $7(36,8)$ \\
Realización personal & $5(26,3)$ \\
\hline
\end{tabular}

como un alto nivel de síndrome de Burnout evaluado al cierre de la unidad.

Respecto a las limitaciones de este estudio es importante recalcar que es un estudio de tipo observacional, limitado al ámbito descriptivo, por lo que no pueden establecerse asociaciones causales. Respecto a la prevalencia de síndrome de Burnout, podría existir un sesgo de representatividad, ya que la encuesta pudo haber sido respondida por las personas que tenían efectivamente presencia de síntomas. Por último, el tiempo de funcionamiento de la unidad acotado a la demanda extraordinaria, no permitió evaluar resultados a largo plazo.

En conclusión, esperamos transmitir la viabilidad de una experiencia como esta, mostrando los complejos y numerosos factores a considerar, los cuales bien gestionados permiten obtener buenos resultados clínicos, a expensas de costos en el bienestar del equipo de salud, lo cual es deseable sea siempre considerado para poder establecer adecuadas y oportunas estrategias de mitigación.

\section{Referencias}

1. Red Hospital Clínico Universidad de Chile [Internet]. Misión- Hospital Clínico Universidad de Chile. 2021 [Cited 2021 Apr 21]; [about 1 screen]. Available from: https://www.redclinica.cl/institucional/mision.aspx

2. Ministerio de Salud de Chile. Diario Oficial de la República de Chile, 2020. DISPONE MEDIDAS SANITARIAS QUE INDICA POR BROTE DE COVID-19. [Internet] (42615), pp.1-6. [Cited 2021 Jan 29] Available from: <https://www.minsal.cl/wp-content/ uploads/2020/03/1745861_web.pdf>

3. WHO-China Joint Mission. Report of the WHO-China Joint Mission on Coronavirus Disease 2019 (COVID-19). Geneva, Switzerland: WHO, Feb; 2020.[Available from: https://digitallibrary. un.org/record/3859867?!n=es].

4. Remuzzi A, Remuzzi G. COVID-19 and Italy: what next? Lancet. 2020 Apr;395(10231):1225-8. https://doi.org/10.1016/S01406736(20)30627-9 PMID:32178769

5. Grasselli G, Pesenti A, Cecconi M. Critical care utilization for the COVID-19 outbreak in Lombardy, Italy: Early experience and forecast during an emergency response: Early experience and forecast during an emergency response. JAMA. 2020 Apr;323(16):15456. https://doi.org/10.1001/jama.2020.4031 PMID:32167538

6. Morawska L, Milton DK. It is time to address airborne transmission of Coronavirus disease 2019 (COVID-19). Clin Infect Dis. 2020 Dec;71(9):2311-3. https://doi.org/10.1093/cid/ciaa939 PMID:32628269

7. World Health Organizatión [Internet]. Transmissión of SARSCoV-2: implications for infection prevention precautions; 2020 July 9 [cited 2021 Apr 21]; [About 1 screen] Available from: 
https://www.who.int/news-room/commentaries/detail/transmission-of-sars-cov-2-implications-for-infection-prevention-precautions

8. NHS. NHS England [Internet]. About coronavirus (COVID-19); c 2020 jan - 2021 Mar [cited 2021 Apr 21]; [About 2 screens]. Available from: https://www.england.nhs.uk/coronavirus/primarycare/about-covid-19/

9. Centers for Disease Control and Prevention [Internet]. COVID-19: SARS-CoV-2 and Potential Airborne Transmission; 2020 Oct 5 [cited 2021 Apr 21]; [about 5 screens]. Available from: https:// www.cdc.gov/coronavirus/2019-ncov/science/science-briefs/ scientific-brief-sars-cov-2.html

10. Meyerowitz EA, Richterman A, Gandhi RT, Sax PE. Transmission of SARS-CoV-2: A review of viral, host, and environmental factors. Ann Intern Med. 2021 Jan;174(1):69-79. https://doi. org/10.7326/M20-5008 PMID:32941052

11. Asadi S, Wexler AS, Cappa CD, Barreda S, Bouvier NM, Ristenpart WD. Aerosol emission and superemission during human speech increase with voice loudness. Sci Rep. 2019 Feb;9(1):2348. https://doi.org/10.1038/s41598-019-38808-z PMID:30787335

12. Meiksin A. Dynamics of COVID-19 transmission including indirect transmission mechanisms: a mathematical analysis. Epidemiol Infect. 2020 Oct;148 e257:e257. https://doi.org/10.1017/ S0950268820002563 PMID:33092672

13. Zhang $R$, Li Y, Zhang AL, Wang Y, Molina MJ. Identifying airborne transmission as the dominant route for the spread of COVID-19. Proc Natl Acad Sci USA. 2020 Jun;117(26):14857-63. https://doi.org/10.1073/pnas.2009637117 PMID:32527856

14. Guan WJ, Ni ZY, Hu Y, Liang WH, Ou CQ, He JX, et al.; China Medical Treatment Expert Group for Covid-19. Clinical characteristics of Coronavirus disease 2019 in China. N Engl J Med. 2020 Apr;382(18):1708-20. https://doi.org/10.1056/NEJMoa2002032 PMID:32109013

15. Mittal R, Ni R, Seo JH. The flow physics of COVID-19 [Internet]. J Fluid Mech. 2020;894 F2:F2. https://doi.org/10.1017/ jfm.2020.330.

16. Netz RR, Eaton WA. Physics of virus transmission by speaking droplets. Proc Natl Acad Sci USA. 2020 Oct;117(41):25209-11. https://doi.org/10.1073/pnas.2011889117 PMID:32973098

17. Rosti ME, Olivieri S, Cavaiola M, Seminara A, Mazzino A. Fluid dynamics of COVID-19 airborne infection suggests urgent data for a scientific design of social distancing. Sci Rep. 2020 Dec;10(1):22426. https://doi.org/10.1038/s41598-020-80078-7 PMID:33380739

18. Cook TM. Personal protective equipment during the coronavirus disease (COVID) 2019 pandemic - a narrative review. Anaesthesia. 2020 Jul;75(7):920-7. https://doi.org/10.1111/anae.15071 PMID:32246849

19. Tran K, Cimon K, Severn M, Pessoa-Silva CL, Conly J. Aerosol generating procedures and risk of transmission of acute respiratory infections to healthcare workers: a systematic review. PLoS One. 2012;7(4):e35797. https://doi.org/10.1371/journal. pone.0035797 PMID:22563403

20. Lim ZJ, Subramaniam A, Ponnapa Reddy M, Blecher G, Kadam
U, Afroz A, et al. Case Fatality Rates for Patients with COVID-19 Requiring Invasive Mechanical Ventilation. A Meta-analysis. Am J Respir Crit Care Med. 2021 Jan;203(1):54-66. https://doi. org/10.1164/rccm.202006-2405OC PMID:33119402

21. Armstrong RA, Kane AD, Kursumovic E, Oglesby FC, Cook TM. Mortality in patients admitted to intensive care with COVID-19: an updated systematic review and meta-analysis of observational studies. Anaesthesia. 2021 Apr;76(4):537-48. https://doi. org/10.1111/anae.15425 PMID:33525063

22. Chang R, Elhusseiny KM, Yeh YC, Sun WZ. COVID-19 ICU and mechanical ventilation patient characteristics and outcomes-A systematic review and meta-analysis. PLoS One. 2021 Feb;16(2):e0246318. https://doi.org/10.1371/journal. pone.0246318 PMID:33571301

23. Araujo, M; Ossandón, P; Abarca, A; Menjiba, A; Muñoz, A. (2021). Pronóstico de pacientes hospitalizados por COVID-19 en un centro terciario en Chile: estudio de cohorte. Medwave; 20(10)30-11-2020.

24. Mostafa A, Kandil S, El-Sayed MH, Girgis S, Hafez H, Yosef M, et al. SARS-CoV-2 seroconversion among 4040 Egyptian healthcare workers in 12 resource-limited healthcare facilities: A prospective cohort study [Internet]. Int J Infect Dis. 2021 Mar;104:534-42. https://doi.org/10.1016/j.ijid.2021.01.037 PMID:33484863

25. Rashid-Abdi M, Krifors A, Sälléber A, Eriksson J, Månsson E. Low rate of COVID-19 seroconversion in health-care workers at a Department of Infectious Diseases in Sweden during the later phase of the first wave; a prospective longitudinal seroepidemiological study. Infect Dis (Lond). 2020;x:1-7. PMID:33232190

26. Chen $Y$, Tong $X$, Wang J, Huang $W$, Yin $S$, Huang $R$, et al. High SARS-CoV-2 antibody prevalence among healthcare workers exposed to COVID-19 patients. J Infect. 2020 Sep;81(3):420-6. https://doi.org/10.1016/j.jinf.2020.05.067 PMID:32504745

27. Fusco FM, Pisaturo M, lodice $V$, Bellopede R, Tambaro O, ParreIla G, et al. COVID-19 among healthcare workers in a specialist infectious diseases setting in Naples, Southern Italy: results of a cross-sectional surveillance study. J Hosp Infect. 2020 Aug;105(4):596-600. https://doi.org/10.1016/j.jhin.2020.06.021 PMID:32565367

28. Alajmi J, Jeremijenko AM, Abraham JC, Alishaq M, Concepcion EG, Butt AA, et al. COVID-19 infection among healthcare workers in a national healthcare system: the Qatar experience. Int J Infect Dis. 2020 Nov;100:386-9. https://doi.org/10.1016/j. ijid.2020.09.027 PMID:32949777

29. Piccoli L, Ferrari P, Piumatti G, Jovic S, Rodriguez BF, Mele F, et al. Risk assessment and seroprevalence of SARS-CoV-2 infection in healthcare workers of COVID-19 and non-COVID-19 hospitals in Southern Switzerland. The Lancet Regional Health - Europe. 2020;(100013):100013.

30. Gómez-Ochoa SA, Franco OH, Rojas LZ, Raguindin PF, Roa-Díaz ZM, Wyssmann BM, et al. COVID-19 in health-care workers: A living systematic review and meta-analysis of prevalence, risk factors, clinical characteristics, and outcomes. Am J Epidemiol. 2021 Jan;190(1):161-75. https://doi.org/10.1093/aje/kwaa191 PMID:32870978 